\title{
SORÇÃO-DESSORÇÃO DO FUNGICIDA CLOROTALONIL EM SOLOS COM DIFERENTES TEORES DE MATÉ RIA ORGÂNICA ${ }^{(1)}$
}

\author{
J . B. REGITANO(2), F. PRATA ${ }^{(3)}$, N. M. P. DIAS ${ }^{(4)}$, \\ A. LAVORE NTI ${ }^{(5)} \&$ V. L. TORNISIELO ${ }^{(2)}$
}

\begin{abstract}
RESUMO
A cinética e a sorção de pesticidas em solos permitem predizer a velocidade com que esta reação atinge o equilíbrio e investigar os possíveis mecanismos envolvidos durante a reação. Portanto, esses processos são fundamentais para que se possa compreender o destino dos pesticidas no solo. Este trabalho teve por objetivo estudar a sorção e a cinética do fungicida clorotalonil em cinco solos com diferentes teores de matéria orgânica do estado de São Paulo: Neossolo Quartzarênico órtico (RQo), Latossolo Vermel ho distroférrico (L Vdf-1 e L Vdf-2), Latossolo Vermelho perférrico (L Vj) e Gleissolo (G). Nestes estudos, foi utilizado traçador radioativo, ou seja, ${ }^{14} \mathrm{C}$-clorotalonil, e a radioatividade foi detectada por espectrometria de cintilação líquida. Os ensaios foram realizados em sala climatizada $\left(25 \pm 2^{\circ} \mathrm{C}\right)$, em ambiente escuro. $O$ ensaio de cinética constou de oito períodos de equilíbrio: $0 ; 0,5 ; 1,0 ; 2,0 ; 4,0 ; 8,0 ; 12,0$ e 24,0 $h$; sendo que nas amostras que atingiram equilíbrio ( $24 \mathrm{~h}$ ) foram realizados os testes de dessorção, em quatro etapas subseqüentes. Nos estudos de isotermas de sorção, as concentrações de clorotalonil empregadas situaram-se entre 0,05 e $0,76 \mu \mathrm{g} \mathrm{mL}^{-1}$. 0 modelo matemático de Elovich foi ajustado aos resultados do estudo de cinética e o modelo de Freundlich foi ajustado aos resultados do estudo de isoterma de sorção. Observou-se elevada sorção de clorotalonil nos solos estudados, exceto no solo arenoso com baixo teor de matéria orgânica (RQo). A sorção do clorotalonil relacionou-se positi vamente com a matéria orgânica do solo. A cinética de sorção desse fungicida envolveu duas fases, uma imediata, de maior relevância quantitativa, e outra mais lenta. Esses resultados mostram que uma pequena fração do clorotalonil aplicado ao solo estaria disponível para ser lixiviado ao lençol freático, mas atenção especial é necessária quando ele é aplicado em solos arenosos com baixo teor de matéria orgânica. Além do que, a fração do fungi cida sorvida na fase lenta também pode ser facilmente dessorvida à solução do solo.
\end{abstract}

Termos de indexação: cinética, pesticida, resíduo ligado, organoclorados, retenção.

\footnotetext{
(1) Recebido para publicação em março de 2001 e aprovado em agosto de 2001.

(2) Pesquisador Científico do CENA, Universidade deSão Paulo- USP. Av. Centenário 303, Caixa Postal 96, CEP 13400-970 Piracicaba (SP). E-mail: regitano@cena.usp.br

(3) Doutorando em Solos e Nutrição de Plantas da Escola Superior de Agricultura Luiz de Queiroz - ESALQ/USP. Bolsista FAPESP.

(4) Doutoranda em Ciências, CENA/USP.

(5) Professor Associado do Departamento de Ciências Exatas, ESALQ/USP.
} 


\title{
SUMMARY: SORPTION-DESORPTION OF CHLOROTHALONIL IN SOILS WITH DIFFERENT ORGANIC MATTER CONTENTS
}

\begin{abstract}
Pesticide kinetics and sorption allow to predict the equilibrium time of the reaction and to speculate over the reaction mechanisms involved. Therefore, their study is fundamental to understand the envi ronmental fate of pesticides. The aim of this research was to evaluatethesorption and kinetics of thefungicidechlorothal onil in fivedistinct soils with different organic matter contents of the state of São Paulo (Brazil): Neossolo Quartzarênico órtico (RQo), Latossol o Verme ho distroférrico (LVdf-1 eLVdf-2), Latossolo Vermelho perférrico (LVj), and Gleissolo (G). The experiments were performed with a radiolabeled tracer, i.e, ${ }^{14} \mathrm{C}$-chlorothalonil, with radioactivity being measured by liquid scintillation spectrometry. The experiments were carried out under controlled laboratory conditions $\left(25 \pm 2^{\circ} \mathrm{C}\right)$, in a dark room. Kinetics was evaluated for 8 equilibrium periods: 0 ; $0.5 ; 1.0 ; 2.0 ; 4.0 ; 8.0 ; 12.0$ and $24.0 \mathrm{~h}$. Desorption was performed in the samples equilibrated for 24 hours, in 4 steps. For sorption isotherm studies, theconcentrations of chlorothal onil ranged from 0.05 to $0.76 \mu \mathrm{g} \mathrm{mL}^{-1}$. The Elovich equation was fitted to the kinetics data whereas theF reundlich equation was fitted to thesorption isotherm studies. Chl orothal onil was highly sorbed to soils, except for the sandy soil with low organic matter content (RQo). Sorption was positively related to soil organic matter content. Moreover, sorption reaction occurred in two phases: a very fast phase occurring in the first few minutes responsible for most of the sorption, followed by a slow phase. The results show that a small fraction of the applied chlorothalonil would be available for groundwater leaching, but special attention is needed when it is applied to sandy soils with low organic matter content. Furthermore, chl orothal onil sorbed during thesl ow phase may beeasily desorbed to the soil solution.
\end{abstract}

Index terms: kinetics, pesticide, bound residues, organochl orines, retention.

\section{NTRODUÇÃO}

Clorotal onil (2,4,5,6-tetradoro-1,3-benzenodicarbonitrilo) é um fungicida não-sistêmico, de largo espectro, que vem sendo intensivamente utilizado em todo o mundo há mais de 30 anos. No Brasil, é indicado para diversas culturas ol erícolas, frutíferas e ornamentais, além de al guns tipos de grãos, como o feijão ea soja (Compêndio de Defensivos Agrícolas, 1993). Segundo Cox (1997), este fungicida é o segundo mais utilizado nos Estados U nidos, com cerca de 5 milhões de kg aplicados anual mente. Ele também figura entre os mais utilizados no Brasil, com um volume de vendas anual de 1,6 milhão de $\mathrm{kg}$, o que representa um mercado de 16 milhões de dólares (comunicado pessoal). Seu principal mecanismo de ação envolve reações com grupos sulfidrilos e glutationas presentes em proteínas e cofatores de fungos (Roberts et al., 1999).

O dorotalonil éum representantedo grupo químico dos organoclorados. Geralmente, as moléculas pertencentes a esse grupo apresentam alta persistência e elevada toxidez, sobretudo para os mamífer os (Montgomery, 1997), sendo consideradas como poluentes em potencial ao ambiente.

O clorotalonil, entretanto, é considerado pouco persistente no ambiente, apresentando valores de meia-vida de dissi pação que variam de 5 a 36 dias
(Sun et al., 1985; Sato \& Tanaka, 1987; Walker et al., 1988; Takagi et al., 1991; Katayama et al., 1997; Motonaga et al., 1998; van der Pas et al., 1999), reflexo da rápida transformação mi crobi ológica eda el evada formação de resíduos ligados, resultante da alta taxa de sorção dessa molécula (Regitano et al., 2001).

Por outro lado, o clorotal onil é contaminado com hexaclorobenzeno durante o processo de síntese industrial, molécula esta queapresenta características carcinogênicas (Cox, 1997). Além disso, os principais metabólitos resultantes da degradação do dorotalonil apresentam menor valor de dose letal média $\left(\mathrm{DL}_{50}\right)$ e maior potencial de lixiviação do que a molécula original e, portanto, têm sido bastante estudados (Sato \& Tanaka, 1987; Rouchaud et al., 1988; Katayama et al., 1991; Cox, 1997). Todas essas características despertam um interesse especial em elucidar o comportamento ambiental dessa molécula, principalmente em solos sob clima tropical, onde poucos experimentos foram realizados com esse fungicida.

Em solos de regiões temperadas, Reduker et al. (1988) e Kawamoto \& Urano (1989) observaram el evados valores de coeficientes de sorção em solos argil osos com teores de matéria orgânica superiores a $2 \%$, ao passo que Gamble et al. (2000) observaram baixa retenção dessa molécula em solos arenosos. 
O objetivo desta pesquisa foi verificar os processos de sorção e dessorção do clorotalonil e sua cinética deligação em sol os com diferentes teores de matéria orgânica, empregando a técnica com traçador radioativo $\left({ }^{14} \mathrm{C}\right)$ da molécula.

\section{MATERIAL E MÉTODOS}

Os ensaios foram realizados no CentrodeE nergia Nuclear da Agricultura da USP, em Piracicaba (SP). Amostras de cinco sol os foram col etadas na camada de $0-20 \mathrm{~cm}$ de profundidade, os quais foram classificados como Neossolo Quartzarênico órtico (RQo), Latossolo Vermelho distroférrico (LVdf-1 e LVdf-2), Latossolo Vermelho perférrico (LVj) e Gleissolo (G), procedentes dos municípios paulistas deSão Pedro, Rio Claroe Piracicaba (os três últimos solos), respectivamente. Estes sol os foram escol hidos por apresentar variações nos seu atributos físicoquímicos que podem influenciar a sorção do clorotal onil. As amostras de sol o foram secas ao ar e passadas em peneira com malha de $2 \mathrm{~mm}$, sendo a umidade residual determinada por gravimetria. As caracterizações quími cas egranulométricas dos solos foram efetuadas de acordo com Raij \& Quaggio (1983) e Camargo et al. (1986) (Quadro 1).

No preparo das soluções de trabalho, foi utilizado o traçador radioativo ${ }^{14} \mathrm{C}$-clorotal onil (marcado uniformemente nos carbonos do anel), cujas características encontram-se descritas na figura 1.

Para estudar a cinética de sorção, amostras de 2,00 g de sol o secos ao ar e $10 \mathrm{~mL}$ de sol ução de ${ }^{14} \mathrm{C}$ clorotalonil na concentração de $0,42 \mathrm{mg} \mathrm{L}^{-1}$ (ou $0,32 \mathrm{kBq} \mathrm{mL}^{-1}$ ), preparada em $\mathrm{CaCl}_{2} 0,005 \mathrm{~mol} \mathrm{~L}^{-1}$, foram adicionadas aos tubos de centrífuga de vidro (50 mL). Logo após, esses tubos foram acondi cionados em agitador horizontal a $120 \mathrm{rpm}$. Posteriormente, em períodos preestabel ecidos $(0 ; 0,5 ; 1 ; 2 ; 4 ; 8 ; 12$ e $24 \mathrm{~h}$ após a aplicação do fungicida), alíquotas de $200 \mu \mathrm{L}$ do sobrenadante foram retiradas (após centrifugação a 3.000 rpm, por $10 \mathrm{~min}$ ); com o objetivo de determinar a concentração de cl orotalonil em sol ução por espectrometria de cintilação líquida $(E C L)$.
A relação solo:solvente e os diferentes períodos de agitação foram estabelecidos em testes preliminares (resultados não exi bidos). O ensaiofoi realizadoem triplicata, inclusive da prova em branco (frascos com solução de cl orotal onil, sem os solos), em sala escura, climatizada a $25 \pm 2{ }^{\circ} \mathrm{C}$. As proporções sorvidas do fungicida foram calculadas por diferença entre o total aplicado e o determinado nas soluções de equilíbrio.

No final, o modelo de Elovich (Sparks, 1989) foi aplicado aos resultados do estudo de cinética:

$$
\mathrm{dq} / \mathrm{dt}=\mathrm{Xe} \mathrm{e}^{\mathrm{Yq}}
$$

em que $=$ quantidade relativa sorvida (\%); $\mathrm{t}=$ tempo de equilíbrio (min); $X$ eY = constantes da equação, específicas ao experimento. A forma integrada desse model o ajustada aos dados destetrabal ho encontrase abaixo:

$$
\hat{\mathrm{q}}=(1 / Y) \ln (X Y)+(1 / Y) \ln \mathrm{t}
$$

em que $[(1 / Y)$ In $(X Y)]$ e $(1 / Y)$ representam o total sorvido no tempo de equilíbrio inicial (fase rápida da cinética de sorção) e a taxa de variação da sorção em função do tempo para a fase lenta da cinética, respectivamente. Estudos de análise de variância foram efetuados para esses parâmetros, sendo calculados os valores das diferenças mínimas significativas pel o testeTukey $(p<0,05)$.

Após o último período de equilíbrio do estudo de cinética (24 h), iniciou-se o estudo de dessorção.

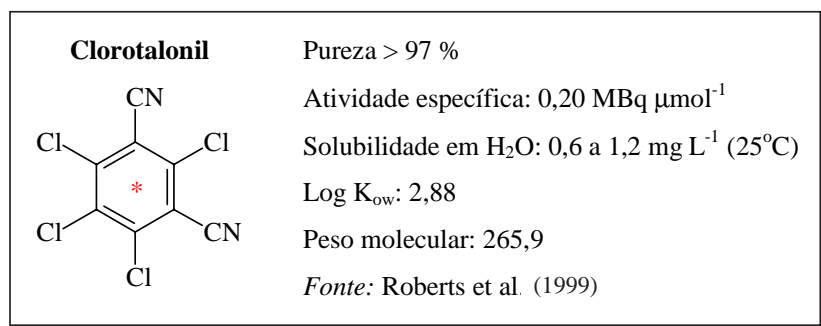

Figura 1. Características físico-químicas do clorotalonil.

Quadro 1. Caracterização química e granulométrica do Neossolo Quartzarênico órtico (RQo), Latossolo

\begin{tabular}{|c|c|c|c|c|c|c|c|c|c|c|c|c|}
\hline Solo & $\mathbf{p H}-\mathrm{H}_{2} \mathrm{O}$ & M.O. & $\mathbf{P}$ & $\mathbf{K}^{+}$ & $\mathrm{Ca}^{2+}$ & $\mathbf{M g}^{2+}$ & $\mathrm{Al}^{3+}$ & $\mathbf{H}+\mathbf{A l}$ & $\mathbf{T}$ & Areia & Silte & Argila \\
\hline & & $\mathrm{g} \mathrm{dm}^{-3}$ & $\mathrm{mg} \mathrm{dm}^{-3}$ & \multicolumn{6}{|c|}{$\mathrm{mmol}_{\mathrm{c}} \mathrm{dm}^{-3}$} & \multicolumn{3}{|c|}{$\mathrm{g} \mathrm{kg}^{-1}$} \\
\hline RQo & 4,5 & 5 & 3 & 0,2 & 4 & 1 & 5 & 20 & 25,2 & 940 & 20 & 40 \\
\hline LVdf-1 & 6,9 & 28 & 39 & 4,3 & 103 & 13 & 0 & 8 & 128,3 & 60 & 100 & 840 \\
\hline LVdf-2 & 4,4 & 20 & 8 & 1,6 & 11 & 5 & 14 & 65 & 82,6 & 220 & 110 & 670 \\
\hline $\mathrm{LVj}$ & 5,1 & 28 & 19 & 1,5 & 30 & 11 & 2 & 45 & 87,5 & 540 & 80 & 380 \\
\hline $\mathrm{G}$ & 5,1 & 150 & 24 & 3,1 & 64 & 15 & 13 & 130 & 212,1 & 180 & 170 & 650 \\
\hline
\end{tabular}
Vermelho distroférrico (LVdf-1 e LVdf-2), Latossolo Vermelho perférrico (LVj) e Gleissolo (G) 
Inicialmente, os sobrenadantes foram descartados, e alíquotas de $15 \mathrm{~mL}$ da solução de $\mathrm{CaCl}_{2}$ 0,005 mol L-1 foram adicionadas aos tubos, os quais for am novamente submetidos à agitaçãoa $140 \mathrm{rpm}$, durante $2,5 \mathrm{~h}$. A pós este período, foram feitas a centrifugação ea retirada de uma alíquota de $1,0 \mathrm{~mL}$ do sobrenadante nos mesmos mol des do estudo de cinética de sorção. Desta forma, a concentração de clorotalonil dessorvida em solução foi determinada por $E C L$. Esse procedimento foi repetido por mais três vezes emtodos os solos. Posteriormente, osoloremanescente foi seco ao ar, destorroado e pulverizado em gral de porcelana, de onde foram retiradas subamostras $(0,40 \mathrm{~g})$, em triplicata, para determinar a concentração sorvida, via combustão em um oxidador biológico. A radioatividade remanescente no sol o foi determinada por $E C L$, o que permitiu o fechamento do balanço da radioatividade.

O procedimento utilizado no estudo de sorção foi semelhante ao de cinética do clorotalonil. No entanto, foram utilizadas cinco concentrações do fungicida: 0,$05 ; 0,09 ; 0,20 ; 0,38$ e $0,76 \mu \mathrm{g} \mathrm{mL}^{-1}$, na proporção de $1,00 \mathrm{~g}$ de solo seco ao ar para 5,0 mL de solução de clorotalonil. O tempo de agitação adotado foi de $8 \mathrm{~h}$, determinado com base no estudo de cinética e na rápida dissi pação da molécula. Para cada concentração, foram efetuadas provas em branco (frascos com solução de clorotalonil, sem os solos). O ensai o foi efetuado em triplicata, sendo a radioatividade determinada por ECL, em alíquotas de $0,5 \mathrm{~mL}$. Para calcular as constantes de sorção, foi utilizado o modelo matemático linearizado de Freundlich (Equação 3).

$$
\log \mathrm{S}=\log \mathrm{K}_{\mathrm{f}}+\mathrm{N} \log \mathrm{C}_{\mathrm{e}}
$$

em queS = concentração da molécula sorvida $\left(\mu \mathrm{g} \mathrm{g}^{1}\right)$; $\mathrm{C}_{\mathrm{e}}=$ concentração da molécula na solução de equilíbrio $\left(\mu \mathrm{g} \mathrm{mL}^{-1}\right) ; \mathrm{K}_{\mathrm{f}}=$ constante de Freundlich $\mathrm{e}$ $\mathrm{N}=$ grau de linearidade da isoterma.

\section{RESULTADOS E DISCUSSÃO}

Recentemente, Gamble et al. (2000) e Regitano et al. (2001) observaram que a formação de resíduos ligados de clorotal onil ocorreu em duas fases: uma fase rápida, nas primeiras $24 \mathrm{~h}$, seguida de uma fase lenta. O fato de os resultados deste trabal ho terem ajustado adequadamente ao modelo de Elovich (Figura 2) indicou que a reação de sorção do dorotalonil também ocorreu em duas fases distintas.

Nestetrabal ho, entretanto, a fase rápida ocorreu nos primeiros minutos de contato do fungicida com os solos (Figura 2), provavelmente, em virtude da diferente concepção metodol ógica desse experimento, principalmente no que se refere à maior exposição dos sítios de retenção dos sol os ao fungicida, a qual foi favorecida pela el evada relação solo:solução

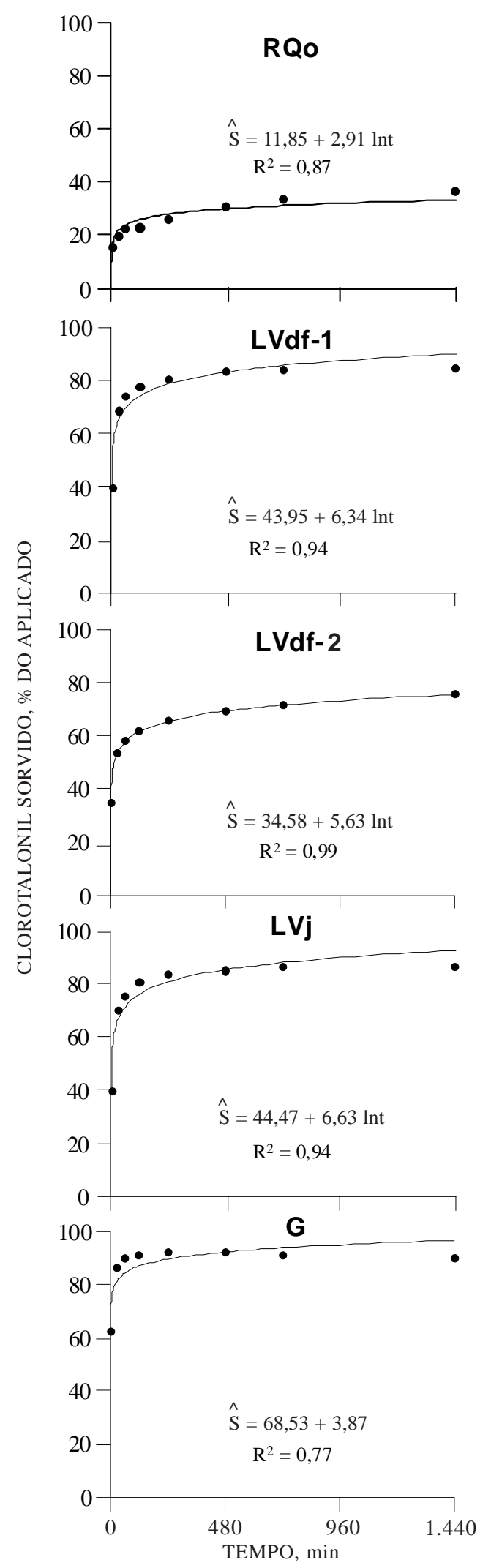

Figura 2. Modelo de Elovich aplicado aos dados de cinética de sorção de clorotalonil no: Neossolo Quartzarênico órtico (RQo), Latossolo Vermelho distroférrico (LVdf-1 e LVdf-2), Latossolo Vermelho perférrico (L Vj) e Gleissolo (G). 
(2,00 g:10,0 mL ) e pel o processo de agitação dos solos. Para estudos de sorção com clorotalonil, o tempo de equilíbrio deve ser o mais breve possível, considerando a rápida transformação microbiana da molécula ( $\mathrm{t}_{1 / 2}=4,1$ dias, Roberts et al., 1999).

As percentagens de clorotalonil sorvidas instantaneamente (na fase rápida), as quais foram descritas pelo valor $[1 / Y$ In $(X Y)]$ do modelo de Elovich, representaram frações significativas da quantidade de fungicida aplicada aos solos (Quadro 2), principalmente naqueles com maiores teores de matéria orgânica (M.O.). Essas frações corresponderam a $76 \%$ da quantidade de clorotanil sorvida no solo G (com maior conteúdo de M.O.) ea $33 \%$ da quantidade de cl orotanil sorvida no sol o RQo (com baixo conteúdo de M.O. e argila). Valores de 52,46 e $51 \%$ foram obtidos para os solos LVdf-1, LVdf-2 eLVj, respectivamente. Resultados semelhantes foram obtidos por Regitano et al. (2001), os quais inferiram que uma pequena quantidade de sítios sortivos estariam disponíveis para que ocorresse rápida ligação do clorotalonil em solos com baixo conteúdo de M.O.

Provavel mente, o principal mecanismo envolvido na retenção do clorotalonil refere-se às interações hidrofóbicas entre o fungicida e a M.O. humificada do solo, uma vez que o clorotalonil apresenta baixa solubilidade em água $\left(\mathrm{S}_{\mathrm{w}}=0,6\right.$ a $\left.1,2 \mathrm{mg} \mathrm{L}^{-1}\right)$ e el evado coeficiente de partição octanol :água ( $\log K_{\text {ow }}=2,88$ ) (Roberts et al., 1999). O clorotalonil é um fungicida não-iônico ea forma mais importante de sorção para moléculas não-iônicas é a interação hidrofóbica (Chiou, 1989). Essas ligações devem ocorrer na fase rápida do processo, nas superfícies externas da M.O., uma vez que as percentagens de clorotal onil sorvidas nessa faseforam diretamente proporcionais aos teores de matéria orgânica dos solos $(Z=-13,95+$ 16,78 In M.O., $R^{2}=0,99 * *$, em que $\left.Z=1 / Y \ln (X Y)\right)$.
A liberação de átomos de cloro, entretanto, constituiu evidência direta de formação de ligação covalente entre os clorofenóis e os ácidos húmicos do solo (Dec \& Bollag, 1994). De acordo com esses autores, a formação de ligações covalentes envolveram reações oxidativas, as quais foram mediadas por enzimas microbianas. $\mathrm{O}$ fato de 0 cl orotalonil não ser dissi pado em sol os autodavados (Sato \& Tanaka, 1987; Katayama et al., 1991; Katayama et al., 1997) e o de as enzimas excretadas por vários mi crorganismos comumente encontrados nos solos estarem envolvidas na degradação do clorotalonil (Katayama et al., 1997) reforçam a hi pótese de formação de ligações covalentes entre o dorotal onil ea M.O. dosoloduranteoprocesso desorção.

A cinética de sorção na segunda fase, ou seja, a vel ocidade de sorção do cl orotal onil na fase lenta do processo sortivo (representada pelo valor $(1 / Y)$ do modelo de Elovich) foi mais rápida nos Latossolos (LVj, LVdf-1 e LVdf-2, em ordem decrescente), seguida do G edo RQo, subseqüentemente(Quadro 2). $\mathrm{O}$ val or $(1 / Y)$ correlacionou-se positivamente com a quantidade de clorotalonil sorvida nessa fase em relação ao total aplicado $\left(\mathrm{R}^{2}=0,86^{* *}\right)$. A fase lenta foi responsável por $67 \%$ da quantidade total de clorotalonil sorvido no RQo e por apenas $24 \%$ da quantidadetotal sorvida no G. Portanto, a fase lenta do processo cinético éimportante para solos arenosos com baixo teor de M.O.

Essas diferenças no processo cinético podem ser atribuídas à restrição no contato do cl or otal onil com a quantidade reduzida de M.O. e, ou, com os aspectos qualitativos desta M.O. no solo arenoso, que pode ter menor grau de humificação e, portanto, apresentar sítios hidrofóbicos com diferentes afinidades pelo clorotalonil, quando comparado a solos com maior teor de M.O. Normalmente, solos com maior quantidadedeM.O. são acompanhados de

Quadro 2. Parâmetros cinéti cos da Equação de Elovich e percentagens sorvida e dessorvida de clorotalonil no Neossolo Quartzarênico órtico (RQo), Latossolo Vermel ho distroférrico (LVdf-1 e L Vdf-2), Latossolo Vermelho perférrico (LVj) e Gleissolo (G)

\begin{tabular}{|c|c|c|c|c|c|c|}
\hline \multirow{2}{*}{ Solo } & \multicolumn{3}{|c|}{ Parâmetro de Elovich } & \multirow{2}{*}{ Stotal $^{(2)}$} & \multirow{2}{*}{ S Ienta $^{(3)}$} & \multirow{2}{*}{$D_{\text {sorvido }}{ }^{(4)}$} \\
\hline & $1 / Y \ln (X Y)$ & $1 / Y$ & $\mathbf{R}^{2}$ & & & \\
\hline \multicolumn{4}{|c|}{$\%$} & \multicolumn{3}{|c|}{ - \% } \\
\hline RQo & $11,85 \pm 0,14 d^{(1)}$ & $2,91 \pm 0,059 d$ & 0,87 & $36,06 \pm 0,57 \mathrm{e}$ & $67,13 \pm 1,01 \mathrm{a}$ & $89,69 \pm 0,20 a$ \\
\hline LVdf-1 & $43,95 \pm 0,74 b$ & $6,34 \pm 0,192 a$ & 0,94 & $84,41 \pm 0,53 c$ & $47,93 \pm 0,24 c$ & $40,75 \pm 0,51 c$ \\
\hline LVdf-2 & $34,58 \pm 0,38 c$ & $5,63 \pm 0,120 b$ & 0,99 & $75,54 \pm 1,19 d$ & $54,22 \pm 2,04 b$ & $54,52 \pm 2,58 b$ \\
\hline $\mathrm{LVj}$ & $44,47 \pm 0,34 b$ & $6,63 \pm 0,001 \mathrm{a}$ & 0,94 & $86,43 \pm 1,09 b$ & $48,54 \pm 0,70 \mathrm{c}$ & $34,57 \pm 1,30 d$ \\
\hline G & $68,53 \pm 1,52 a$ & $3,87 \pm 0,615 c$ & 0,77 & $89,91 \pm 0,13 a$ & $23,79 \pm 1,91 \mathrm{~d}$ & $28,72 \pm 1,54 \mathrm{e}$ \\
\hline
\end{tabular}

\footnotetext{
(1) Médias com letras iguais, nas colunas, não diferem estatisticamente pelo teste deTukey $(p<0,05)$. ${ }^{(2)}$ Percentagem sorvida após $24 \mathrm{~h}\left({ }^{14} \mathrm{C}\right.$-clorotalonil aplicado $\left.=100 \%\right)$. (3) Percentagem sorvida na fase lenta em relação ao sorvido $\left({ }^{14} \mathrm{C}\right.$-d orotal onil sorvido $\left.=100 \%\right)$. (4) Percentagem dessorvida $\left({ }^{14} \mathrm{C}\right.$-clorotalonil sorvido $\left.=100 \%\right)$.
} 
uma maior atividade microbiana, razão pela qual apresentam uma M.O. mais humificada, com maior quantidade desítios hidrofóbicos (Martin-N eto et al., 1998).

Gamble et al. (2000) estudaram a cinética de formação de resíduos ligados de ${ }^{14} \mathrm{C}$-cl orotal onil em sol os semel hantes ao RQo. Utilizando modelos diferenciais baseados na Lei de Fick, observaram que $30,5 \%$ do fungi cida aplicado ficou retido no solo após 18 dias da aplicação. Essevalor mostrou-se bastante semel hanteao obtido nestetrabal ho (30,06\%), apesar de el es terem utilizado extratores mais enérgicos.

Considerando as evidências teóricas, esses autores sugeriram que cinética de transferência de massas ocorreu por difusão do clorotalonil para o interior das substâncias húmicas do solo. Segundo Nam \& Alexander (1998), o seqüestro de moléculas orgânicas apolares ocorreu quando estas moléculas penetraram, por difusão, nos nanoporos do sol o com superfícies hidrofóbicas. Para Fuhr (1987), a difusão é um dos principais mecanismos envolvidos na formação deresíduos ligados de pesticidas orgânicos com os solos.

A Lei deFick podeser utilizada para determinar se a sorção de uma molécula é compatível com o processo de difusão. N esse caso, a quantidade total da molécula sorvida deve apresentar correlação linear com o valor da raiz quadrada do tempo de equilíbrio (Leenher \& Alrichs, 1971; Kookana et al., 1992; Gambleet al., 2000). A não-linearidadeinicial das curvas na figura 3 indica que o mecanismo de difusão não é importante na retenção do cl orotal onil na fase rápida. Entretanto, a difusão pode tornarse importante na fase lenta do processo (Figura 3).

Val ores de $K_{f}$ superiores a $5\left[\left(\mathrm{mg} \mathrm{g}^{-1}\right)\left(\mathrm{mL} \mathrm{mg}^{-1}\right)^{\mathrm{N}}\right]$ revelam alta sorção do pesticida no solo (Green \& Karickhoff, 1990). Portanto, a sor ção do cl orotalonil foi elevada em todos os solos, à exceção do RQo (Quadro 3). Valores de $\mathrm{K}_{\mathrm{f}}$ semelhantes aos obtidos

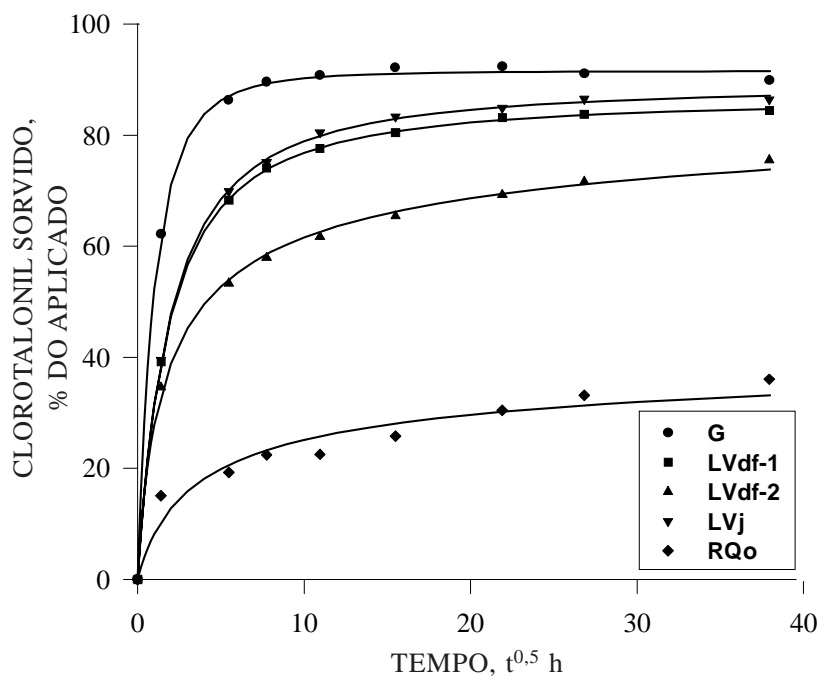

Figura 3. Quantidade de clorotalonil sorvido em função da raiz quadrada do tempo para o Neossolo Quartzarênico órtico (RQo), Latossolo Vermelho di stroférrico (LVdf-1e LVdf-2), Latossolo Vermelho perférrico (L Vj) e Gleissolo (G).

neste trabalho também foram apresentados por Reduker et al. (1988); Kawamoto \& Urano (1989); Motanaga et al. (1998) e Regitano et al. (2001). No entanto, Kawamoto \& Urano (1989) obtiveram valores ainda maiores de $\mathrm{K}_{\mathrm{f}}$, chegando a $150\left[\left(\mathrm{mg} \mathrm{g}^{-1}\right)\right.$

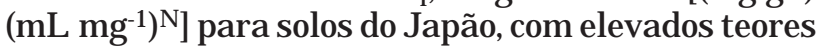
de M.O.

A el evada sor ção do cl orotal onil está associada à fração orgânica do sol o, como podeser observado pela estreita relação linear obtida entre o coeficiente de distribuição $K_{d}\left(K_{d}=K_{f}\right.$, quando $\left.\mathrm{N}=1\right)$ e o teor de carbono orgânico dos solos ( $\mathrm{K}_{\mathrm{d}}=11,20+0,60 \mathrm{C}_{\text {org }}$ $\left.\mathrm{R}^{2}=0,91^{* *}\right)$. Resultados semel hantes encontrados na literatura também demonstram a importância da M.O. do solona retenção doclorotalonil, conferindo papel secundário às argilas.

Quadro 3. Valores do coeficiente de distribuição $\left(K_{d}\right)$, coeficiente normalizado ao teor de $C_{\text {org }}\left(K_{o c}\right)$ e parâmetros de F reundlich para sorção de clorotalonil no Neossolo Quartzarênico órtico (RQo), Latossolo Vermel ho distroférrico (LVdf-1 e LVdf-2), Latossolo Vermelho perférrico (LVj) e Gleissolo (G)

\begin{tabular}{|c|c|c|c|c|c|}
\hline \multirow{3}{*}{ Solo } & \multirow{3}{*}{$\mathbf{K}_{\mathbf{d}}$} & \multirow{3}{*}{$K_{\text {oc }}$} & \multicolumn{3}{|c|}{ Parâmetro de Freundlich } \\
\hline & & & \multicolumn{3}{|c|}{ Sorção } \\
\hline & & & $\mathbf{K}_{\mathbf{f}}$ & $\mathbf{N}$ & $\mathbf{R}^{2}$ \\
\hline & $\longrightarrow$ & $g^{-1}$ & {$\left[\left(\mathrm{mg} \mathrm{g}^{-1}\right)\left(\mathrm{mL} \mathrm{mg}^{-1}\right)^{\mathrm{N}}\right]$} & & \\
\hline RQo & $1,51 \pm 0,02 \mathrm{~d}^{(1)}$ & $301,33 \pm 4,16 b$ & $1,87 \pm 0,68$ & $0,99 \pm 0,09$ & 0,98 \\
\hline LVdf-1 & $31,55 \pm 0,16 b$ & $1126,79 \pm 5,72 \mathrm{a}$ & $52,52 \pm 2,37$ & $0,82 \pm 0,01$ & 0,99 \\
\hline LVdf-2 & $16,24 \pm 0,51 \mathrm{c}$ & $969,83 \pm 274,10 a$ & $42,19 \pm 6,85$ & $0,72 \pm 0,06$ & 0,99 \\
\hline LVj & $26,04 \pm 3,97 b$ & $930,00 \pm 141,82 a$ & $27,63 \pm 2,52$ & $0,72 \pm 0,06$ & 0,99 \\
\hline G & $61,27 \pm 6,14 a$ & $408,27 \pm 40,65 b$ & $55,88 \pm 10,77$ & $1,08 \pm 0,06$ & 0,99 \\
\hline
\end{tabular}

(1) Médias com letras iguais, nas colunas, não diferem estatisticamente pelo teste de Tukey $(p<0,05)$. 
Como o teor de carbono orgânico é o principal atributo do solo relacionado com a sorção de moléculas orgânicas apolares em solos, um índice bastante utilizado em model agem para normalização da sor ção de pesticidas é o $K_{o c}\left(K_{o c}=K_{d} / C_{o r g}\right)$ (Green \& Karickhoff, 1990). Para esses solos, os valores de $\mathrm{K}_{o c}$ encontram-se descritos no quadro 3. Entretanto, sea fração orgânica do sol o fosse a única responsável pela sorção do cl orotalonil, os val ores de $K_{o c}$ para os diferentes sol os deveriam ser iguais, o que não foi o caso (Quadro 3).

Para dez pesticidas organoclorados, Kawamoto \& Urano (1989) obtiveram a seguinte correlação entre $\mathrm{K}_{\mathrm{oc}} \mathrm{eK}_{\mathrm{ow}}: \log \mathrm{K}_{\mathrm{oc}}=0,638 \log \mathrm{K}_{\text {ow }}+1,137$. Para o clorotal onil, que apresenta $\mathrm{K}_{\text {ow }}=437$, o val or $\mathrm{K}_{\mathrm{oc}}$ calculado pela equação deK awamoto \& U rano (1989) é $663 \mathrm{~mL} \mathrm{~g}^{-1}$, o que está dentro da faixa de valores obtidos neste trabal ho (Quadro 3).

Cerca de 28 a $43 \%$ do clorotalonil aplicado foi dessorvido à solução do solo. I sto representou cerca de $29 \%$ da quantidade sorvida no sol o G (com maior teor de M.O.) e cerca de $90 \%$ da quantidade sorvida no solo RQo (com menor teor de M.O. e argila). Valores intermediários foram obtidos para os Latossol os (Quadro 2). Além disso, as percentagens de clorotalonil dessorvidas foram semel hantes aos val ores sorvidos na fase lenta (Quadro 2). I stoindica que somente a fração do clorotalonil sorvida mais fracamente, como exemplo, por difusão aos sítios hidrofóbicos do solo, estaria sendo liberada durante o processo de dessorção.

As interações hidrofóbicas representam sítios específicos de sorção, os quais podem apresentar diferentes afinidades, dependendo dos aspectos qualitativos da M.O. (Celis et al., 1997; Martin-N eto et al., 1998). Portanto, se nenhum mecanismo forte de ligação (por exemplo, as ligações covalentes) estiver envolvido na sorção do clorotalonil, as diferenças no seu potencial de dessorção podem ser explicadas pela baixa efi ciência das soluções sal inas aquosas em extrair o clorotalonil dos sítios hidrofóbi cos nos sol os com mai or quantidade de M.O. Por exemplo, a mai or sorção e, conseqüentemente, a menor dessorção (e maior histerese) dos herbicidas atrazina e simazina de um ácido húmico comercial (AHC) em relação a um ácido húmico extraído do solo (AHS) deveram-se à maior hidrofobicidade do AHC, uma vez que este apresentou maior valor de $\mathrm{pH}$ e ambos apresentaram teores semelhantes de carbono orgânico (Celis et al., 1997).

À exceção do RQo, o clorotal onil foi muito sorvido nos solos, estando sua sorção intimamente relacionada com o teor de M.O. dos solos. Embora isso indique um baixo potencial de lixiviação dessa molécula, a sua lixiviação pode ocorrer em solos arenosos com baixo teor de M.O., devido à menor afinidade do clor otalonil aos sítios sortivos neste tipo de solo. A rápida dissi pação do cl orotal onil em solos é, em grande parte, devida à sua rápida sorção às frações orgânicas do sol o. No entanto, o clorotal onil também pode ser rapidamente biodegradado no sol o a metabólitos muito mais polares e, conseqüentemente, mais solúveis em água e com menor afinidade com a M.O., o que realça o seu potencial poluente. Por exemplo, o 1,3-diciano-4hidroxi-2,5,6-triclorobenzeno é um dos principais metabólitos resultantes da degradação do clorotalonil (Rouchaud et al., 1988; Regitano et al., 2001), com mobilidade muito maior, além de ser 30 vezes mais tóxico quea molécula original (Cox, 1997).

\section{CONCLUSÕES}

1. O cloratalonil é um fungicida que apresenta el evada sorção, a qual está diretamente relacionada com o teor de matéria orgânica do solo.

2. A cinética de sorção do clorotalonil é constituída de duas fases: a fase rápida, que ocorre nos primeiros minutos da reação, seguida da faselenta.

3. Quantitativamente, a fase rápida da reação é mais importante para a sorção do clorotal onil, exceto em solos arenosos com baixo teor de matéria orgânica.

\section{LITERATURA CITADA}

CAMARGO, O.A.; MONIZ, A.C.; J ORGE, J .A. \& VALADARES, J.M. Métodos de análise química, mineralógica e física de solos. Campinas, IAC, 1986. 94p.(Boletim Técnico, 106)

CELIS, R.; CORNEJ O, J.; HERMOSÍN, M.C. \& KOSKINEN, W. Sorption-desorption of atrazine and simazine by model soil colloidal components. Soil. Sci. Soc. Am. J ., 61:436-443, 1997.

CHIOU, C.T. Theoretical considerations of the partition uptake of nonionic organic compounds by soil organic matter. In: SAWHNEY, B.L. \& BROWN, K., eds. Reactions and movement of organic chemicals in soils. Madison, Soil Science Society of America, 1989. p.1-29.

COMPÊNDIO de defensivos agrícolas. 4.ed. São Paulo: Organização Andrei, 1993. 448p.

COX, C. Chlorothalonyl. J. Pest. Reform., 17:14-20, 1997.

DEC, C. \& BOLLAG, J.M. Dehalogenation of chlorinated phenols during oxidative coupling. Environ. Sci. Technol., 28:448490, 1994.

FÜHR, F. Non-extractable pesticides residues in soil. In: GREENHALG, R. \& ROBERTS,T.R., eds. PesticideScience and Biotechnology. Oxford, International Union of Pure and Applied Chemistry, 1987. p.381-389.

GAMBLE,D.S.; BRUCCOLERI,A.G.; LINDSAY, E.; LANGFORD, C.H. \& LEYS, G.A. chlorothalonil in a quartz sand soil: Speciation and kinetics. Environ. Sci. Technol., 34:120124, 2000. 
GREEN, R.E. \& KARICKHOFF, S.W. Sorption estimate for modeling. In: CHENG, H.H., ed. Pesticides in the soil environment: Processes, impacts, and modeling. Madison, Soil Science Society of Americam, 1990. 530p. (Am. Book Series)

KATAYAMA, A.; ISEMURA, H. \& KUWATSUKA, S. Supression of chlorothal onil dissipation in soil by repeated applications. J. Pest. Sci., 16:233-238, 1991.

KATAYAMA, A.; ITOU, T. \& UKAI, T. U biquitous capability to substitute chlorine atoms of chorothalonil in bacteria. J. Pest. Sci., 22:12-16, 1997.

KAWAMOTO, K. \& URANO, K. Parameters for predicting fate of organochlorine pesticides in the environment (II) Adsorption constant to soil. Chemosphere, 19:1223-1231, 1989.

KOOKANA, R.S.; AYLMORE, L.A.G. \& GERRITSE, R.G. Time dependent sorption of pesticides during transport in soils. Soil Sci., 154:214-225, 1992.

LEENHER, J.A. \& AHLRICHS, J.L. A kinetic and equilibrium study of the adsorption of carbaryl and parathion upon soil organic matter surfaces. Soil Sci. Soc. Am. Proc., 35:700704, 1971.

MARTIN-NETO, L.; ROSELL, R. \& SPOSITO, G. Correlation of spectroscopic indicators of humification with mean annual rainfall along a temperate grassland climosequence. Geoderma, 81:305-311, 1998.

MONTGOMERY, J.H. Agrochemicals, desk reference. 2.ed. New York, Lewis Publishers, 1997. p.101-102.

MONTONAGA, K.;TAKAGI, K.\& MATUMOTO, S. Suppression of chlorothalonil degradation in soil after repeated application. Environ. Tox. Chem., 17:1469-1472, 1998.

NAM , K. \& ALEXANDER, M. Role of nanoporosity and hydrophobicity in sequestration and bioavailability: Tests with model solids. Environ. Sci. Technol., 32:71-74, 1998.

RAIJ , B. van \& QUAGGIO, J.A. Métodos de análise de solos para fins de fertilidade. Campinas, Instituto Agronômico de Campinas, 1983. 31p. (Bol etim Técnico, 81)
REDUKER, S.; UCHRIN, C.G., \& WINNETT, G. Characteristics of the sorption of chlorothalonil and azinphos-methyl to a soil from a commercial cranberry bog. Bul. Environ. Contam. Toxicol., 41:633-641, 1988.

REGITANO, J.B.; TORNISIELO, V.L.; LAVORENTI, A. \& PACOVSKY, R.S. Transformation pathways of ${ }^{14} \mathrm{C}$ chlorothalonil in tropical soils. Arch. Environ. Contam. Toxicol., 40:295-302, 2001.

ROBERTS, T.; HUDSON, D.; JEWESS, P.J .; LEE, P.W. \& PLIMMER,J.R. Metabolic pathways of agrochemicals. Part 2:I nsecticides and fungicides. Cambridge. The Royal Society of Chemistry, 1999. p.1380-1384.

ROUCHAUD, J .; ROU COURT, P. \& VANACHTER, A. Hydrolytic biodegradation of chlorothalonil in the soil and in cabbage crops. Toxical. Environ. Chem., 17:59-68, 1988.

SATO, K. \& TANAKA, H. Degradation and metabolism of a fungicide, 2,4,5,6-tetracloroi softalonitrilo (TPN) in soil. Biol. Fertil. Soils, 3:205-209, 1987.

SPARKS, D.L. Kinetics of soil chemical processes. London, Academic Press, 1989. 210p.

SUN, T.; HASHIMOTO, T.; WADA, H. \& TAKAI, Y. Effects of organic amendments on soil microbial ecosystem modified by long-term application of pesticides. Nippon Dojohiryogaku Zasshi, 56:31-36, 1985.

TAKAGI, K.; WADA, H. \& YAMAZAKI, S. Effect of a long term application of a fungicide, chlorothalonil (TPN) on upland ecosystem. Soil Sci. Plant Nutr., 37:583-590, 1991.

VAN DER PAS, L.J.T.; MATSER, A.M.; BOESTEN, J .J.T.I . \& LEISTRA, M. Behavior of metamitron and hydroxychlorothalonil in low-humic sandy soils. Pestic. Sci., 55:923934, 1999

WALKER,W.W.; CRIPE, C.R.;PRITCHARD, P.H.\& BOURQUIN, A.W. Biological and abiotic degradation of xenobiotic compounds in vitro estuarine water and sediment/water systems. Chemosphere, 17:2255-2270, 1988. 